\title{
Method for Signals Detection in Single Crystal Diffraction Patterns through a Diffraction Pattern Indexing Software
}

\author{
Tania Paola Campos Frías ${ }^{1}$, Rafael Vázquez Pérez ${ }^{1}$ and Carlos Elías Ornelas-Gutiérrez ${ }^{2}$ \\ 1. División de Estudios de Posgrado-Departamento de Sistemas y Computación, Instituto Tecnológico de Chihuahua II, Chihuahua \\ 31130, México \\ 2. Centro de Investigación en Materiales Avanzados S.C., Laboratorio Nacional de Nanotecnología, Miguel de Cervantes No. 120 , \\ Chihuahua, Chih. C.P. 311136, Mexico
}

\begin{abstract}
The correct use of information in science and technology is very important for its progress. Nowadays, the equipment used for the scientific and technological development provides results that are later interpreted by the researchers, in most of the above mentioned equipment the results are images full of information which has to be analyzed. A powerful stage with multiple benefits in this field is the image pre-processing by means of intelligent systems, which are capable to do image analysis throwing very useful results that enhance the scientific and technological information. There are currently more than 500 functions in the computational vision specialized open source library OpenCV, which associated with the $\mathrm{C}++$ programming language. These functions are used for application development in many areas of computer vision such as products inspection, medical images, safety, user's interfaces, camera calibration, stereoscopic vision and robotics. In this development and research work, by using the available functions and modifying the exposed methods, we present a proposal for signal detection in images originated in the transmission electron microscope (known as diffraction patterns), which are attached to the detailed analysis of crystalline structures used in the study of the materials science, the results show a profit of at least $18 \%$ in the detection of signs by means of the method proposed in this work.
\end{abstract}

Key words: Diffraction pattern, OpenCV, detection, image pre-processing.

\section{Introduction}

The development of science and technology is related to the knowledge we have of materials; the physical and chemical composition of these materials will determine the potential use or application for them. Crystallography is the science concerned with the development and growth of crystalline materials, their physical and chemical properties, their outward form, their internal structure and the location of their atoms. It has been found that there is an obvious correlation between the crystallinity of the materials and their physico-chemical properties [1, 2].

In order to determine the crystallinity of the material,

Corresponding author: Carlos Elias Ornelas Gutierrez, M.Sc., research fields: nanotechnology and nanostructured materials, high resolution transmission electron microscopy, and catalyst. E-mail: carlos.ornelas@cimav.edu.mx. it is necessary to fully characterize it. The TEM (transmission electron microscope) is one of the most used equipment in technological research and basic and applied science to analyze crystalline samples. This sophisticated microscope utilizes a beam of accelerated electrons to study the thin sample, obtaining a vast amount of information resulting from the interaction of the electron beam with the sample, which is collected by several detectors in the equipment [3].

When electrons impact the sample, a set of signals is generated from which the diffracted electrons are directly related with its atomic structure. This signals of diffracted electrons collected by the detectors, are known as diffraction patterns. These diffraction patterns, in turn, produce various types of images depending on the analyzed sample. Many analytical 
data are obtained from these diffraction patterns, which allow to study the particles chemical composition, microstructural defects in crystalline materials, measure distance, angles, size and radius at a nanometric scale, identify crystalline phases, among others [4-6]. The crystalline phases are the parts of the material which have the same atomic ratio and crystalline arrangement; every phase is a homogeneous element of the total mass, with its specific properties and characteristics [7].

Therefore, in a heterogeneous system with differing atomic ratios or varying crystalline relationships, each one of the uniform elements of which it is composed is separable and distinguishable from each other and they are known as phases. These phases can have the same chemical composition (diamond-graphite) or completely different (graphite-iron carbide) [8].

An accurate characterization of the studied materials enables researchers to analyze their particular properties so that they can improve the performance in a specific use or create new materials for a given application; thus, processing the information contained in images is essential for science and technology development.

Numerous papers have been written about image processing by using the $\mathrm{C}++$ programming language, supported by the open source, computer vision specialized library, OpenCV, which includes over 500 computer vision methods, such as product inspection, medical images, security, user interface, camera calibration, stereoscopic vision and robotics [9].

Image pre-processing is a very common and crucial step in any computer vision system. It incorporates a set of algorithms that are applied to an image to improve its quality [10], some of which can include noise removal and geometric transformations, improving contrast and vividness, etc. [11].

Using the tools included in the OpenCV library for image pre-processing, Xie and Lu suggest a method for detecting the amount of copper in wires [12], Lorsakul and Suthakorn propose a procedure to identify traffic signs [13] and a system to recognize blood cells [14].
Nevertheless, these methods are not capable of detecting all the signals available in the diffraction pattern image, causing the loss of significant information, particularly in the regions furthest from the center due to their adverse light conditions; and even though these signals are not very perceptible visually, they are highly useful for the researchers.

The CIMAV (Centro de Investigación en Materiales Avanzados) has two TEMs, one of which is high resolution (HRTEM) JEOL JEM 2200FS+Cs. Different types of diffraction pattern images of various materials are obtained with these microscopes to determine the phases of the analyzed material; however, the method can include images taken from any microscope.

Around year 2000, Intel Microprocessor Research Lab developed an Open Source Computer Vision Library, better known as OpenCV [15], which is distributed under the BSD License and hence it is free for both academic and commercial use. OpenCV can be used in both Linux and Windows. It also supports a wide variety of other operating systems. It contains an optimized collection of C libraries across a broad range of computer vision algorithms. Some of the function areas endorsed by this library are [16]:

- Basic structures and operations on arrays;

- Image processing and analysis;

- Structural analysis and shape descriptors;

- Motion analysis and object tracking;

- Face recognition and object detection;

- Camera calibration and 3D reconstruction;

- Machine learning;

- Reading and writing video;

- User interface.

This paper presents an image processing approach, which includes a novel method utilizing the OpenCV library and its tools for signals detection in diffraction pattern images. This method is capable of increasing the number of signals detected, providing up to $18 \%$ improvement compared to other methods reported for similar applications. 


\section{Software Approach}

This work utilizes the Qt IDE (integrated development environment) for the design and development of the diffraction pattern detection software, as well as the $\mathrm{C}++$ programming language and the open source library specialized in computer vision called OpenCV [17], which collects over 500 functions typical of this area [9], to suggest a method that is capable of detecting the widest possible number of signals within a crystal diffraction pattern (single crystal). The signals are usually shown as spots in the image; this method is called SIPAD (Spanish acronym of diffraction pattern identification software) and is employed to automatically detect signals in a diffraction pattern image of a single crystal.

The quality of the image needs to be improved by implementing a common stage known as pre-processing [10], which is a collection of algorithms that are applied to an image to modify it and reduce the noise it might have through geometric transformations, intensity and contrast improvement, among other operations.

Some of the most common functions contained in the OpenCV library to process images are [11, 18-20]:

- Thresholding: removes the values that are above or below a certain value called threshold, assigning a zero value to them;

- Binarization: thresholding variant that changes all pixel values to zero when they are below the threshold and to one when they have the same value or greater;

- Dilate: spreads the number of pixels of an object, typically in all directions simultaneously;

- Erosion: shrinks the shape of an object by removing pixels from the edge that defines it;

- Filtering: operation that softens an image through computation and assignment of the average values surrounding each pixel in the image.

The main advantage of using SIPAD is the possibility to semi-automate the analysis and indexing process of the diffraction patterns obtained from TEMs; in other words, this software is capable of proposing the spots that are considered the most relevant to the user, and at the same time allowing modifications if necessary.

\subsection{Pre-processing Algorithms}

By themselves, the methods that are incorporated into the OpenCV library typically cannot detect the entire number of characteristics or signals that are being sought in an image; therefore, are supported by other functions to achieve better outcomes. This is primarily due to the high degree of specialization that each function has; for instance, if border detection of a shape in an image is needed and the latter has not been filtered, it is possible that many edges are detected erroneously because of the noise existing in the image. In this case, the best practice is to apply a filter to the image before implementing the border detection operation.

The method suggested by Xie and Lu [12] to reveal the amount of copper in cables, consists of the following phases:

(1) Image capture;

(2) Grayscale conversion;

(3) Median filter;

(4) Thresholding;

(5) Morphological operations: erosion, dilation, opening;

(6) Edge detection.

In a preliminary approach, the Xie and Lu method [12] applied to a diffraction pattern allows to identify a considerable amount of signals; however, it also detects the information at the bottom of the image, which is considered as noise in this process. This is shown in Fig. 1.

In order not to produce fake signals at the bottom of the image in Fig. 1a, it is necessary to remove some information from the original image, eliminating the detection of non-present signals and causing an improvement of the results. This process is applied to the same image, resulting in the detection as shown in Fig. 2. 


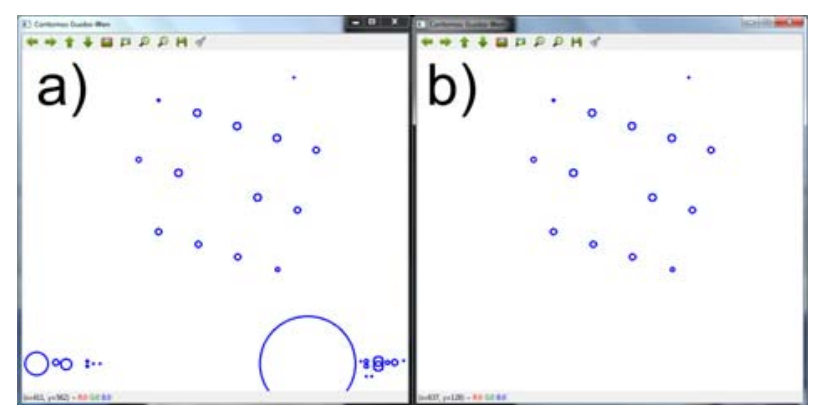

Fig. 1 (a) Signal detection with the Xie and Lu's method applied to the original image and (b) signal detection with the same method removing the bottom information of the image.

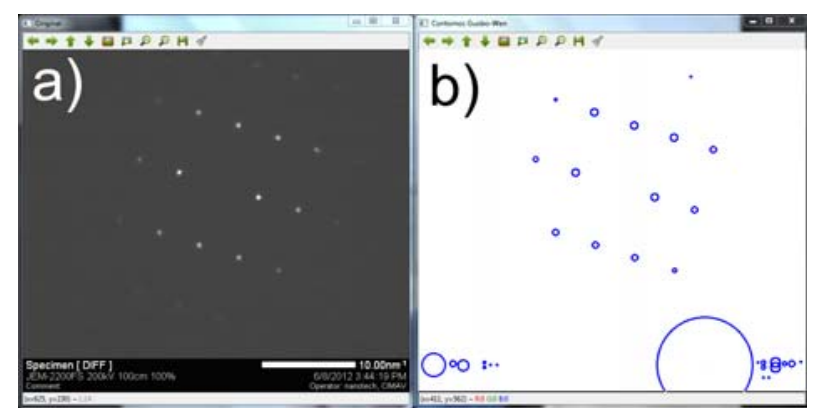

Fig. 2 (a) Original image and (b) edge detection on the image with the Xie and Lu method.

Xie and Lu's method shows good results identifying signals in diffraction patterns images; however, due to the impact that results have in the research area, it is necessary to consider as much signals in the image as possible and supply SIPAD with the ability to identify most of them. It is important to detect the maximum amount of signals, since they all contain important data about the analyzed material, which is very useful and essential for researchers. Not considering this information could impact the studies, resulting in incomplete analysis and thus losing substantial characteristics of the sample from which the diffraction pattern was obtained.

A more detailed review of the original image shows that the signals that are further away from the center of the image, which due to the low light conditions at the borders of the image, are not very perceptible visually. Fig. 3 exhibits the equalization of the original image to show the signals that are not perceivable in the non-equalized original image. Looking at the image, it is noticeable that the method proposed by Xie and $\mathrm{Lu}$ is not capable of detecting all signals, hence resulting in an incomplete detection procedure for this specific application.

There are other methods very similar for particle discovery like Lorsakul and Suthakorn's [13] for traffic signs detection, or Priyankara et al. [14] for blood cells recognition. Nevertheless, these procedures are not capable of detecting the signals of interest that are located further from the center of the image because of the low light condition at the edges which, even when they are hard to see, can be identified.

Fig. 3 also shows that the signals which move away from the center are less intense than the ones closer to it, and even when they are present it is hard for the system to identify them. It can be seen that different spots (signals of interest) in the image have a very similar behavior, regardless of their distance from the center of the image, namely, their intensity. It was found that in every spot, there is a central region composed of one or more pixels, all with the same value (which we will call maximum), from which the intensity of the spot decreases until pixels of the image background are reached. This behavior is demonstrated in Fig. 4.

Based on the intensive analysis, a new method is proposed to increment signals detection in diffraction

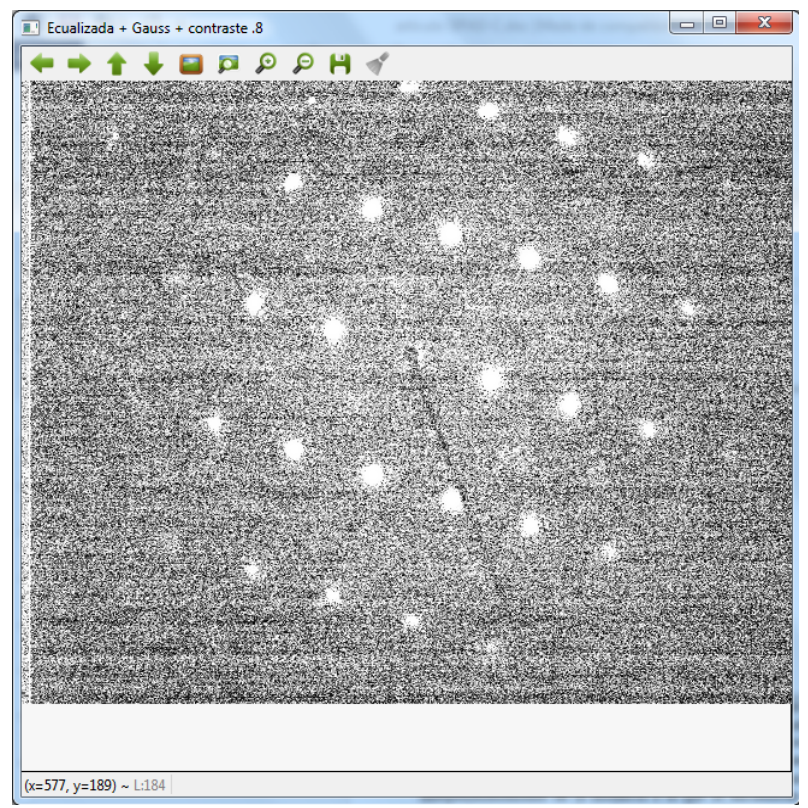

Fig. 3 Original image equalization. 


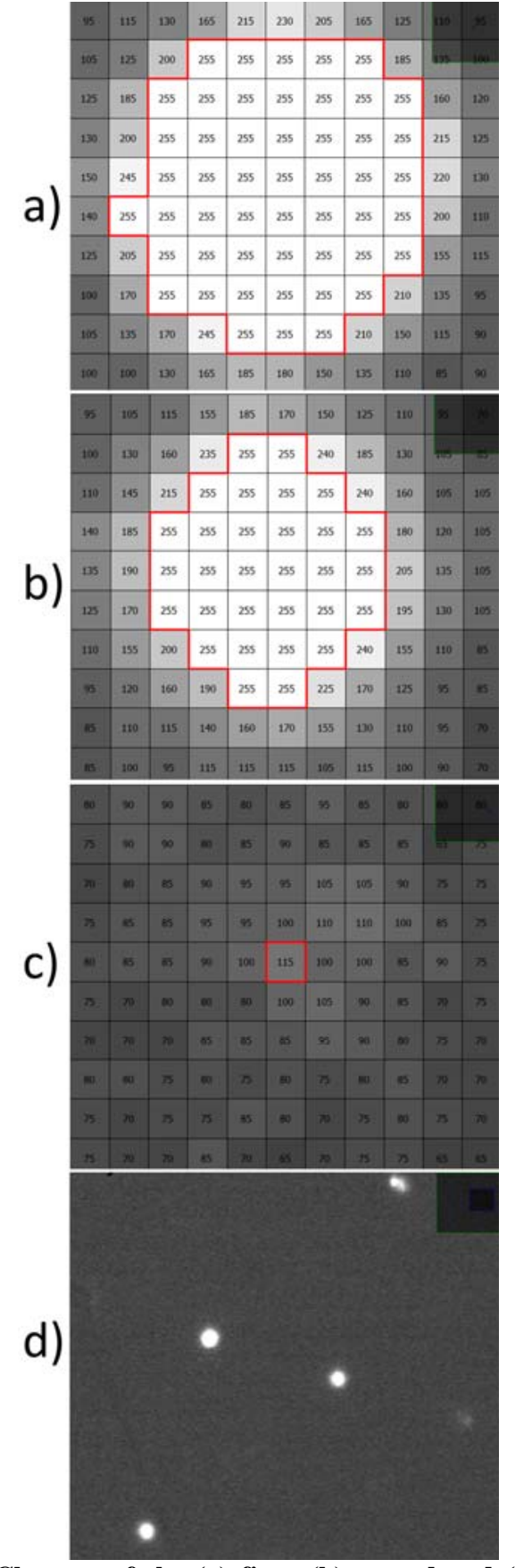

Fig. 4 Close-up of the (a) first, (b) second and (c) third signals to the right of the image center and (d) their location in the image. Outlined in red is the center spot of each signal (50 pixels with a 255 value for the first signal, 30 pixels with a 255 value for the second signal and a single pixel with a value of $\mathbf{1 1 5}$ in the third signal). As the pixels that encircle the signal are further from the center spot, their value decreases.

patterns images. This algorithm consists of the following stages:
(1) Maximum detection: the system needs to discover possible diffraction pattern signals. This is achieved by convolving the image with a $7 \times 7$ kernel; this is, passing a $7 \times 7$ mask through all pixels in the image. For each segment inside the kernel, the mean value is calculated and if there is a pixel which value is slightly higher than the estimated value, it is marked as a maximum since it is probably the centre of a signal of interest;

(2) Spots search: once possible maximum pixels are located, the next step is to verify if they effectively belong to the centre of a signal. This is performed based on the information surrounding the pixel marked as maximum: if the behavior is similar to the one illustrated in Fig. 4, then it is set as the centre of a signal of interest and the pixels surrounding it, considered as a part of the signal are also selected; if these conditions are not met, the maximum that is being analyzed is discarded as a part of a signal. Next, for the final signals detected the dilation, thresholding and erosion functions are applied to the image to remove potential noise added through the process and to acquire a binary image on which signals of interest contours will be detected.

(3) Contours detection: finally, the OpenCV findContours() function is implemented to get the contours of the connected components in an image [21], obtaining the spots as shown in Fig. 5a.

\section{Results}

From the available files, a simple of 21 different diffraction patterns images was selected for the purpose of testing the algorithms described in this work. After applying both techniques, the suggested method revealed a better performance for spot diffraction patterns than the one proposed by Xie and Lu, identifying approximately $18 \%$ more signals. The discrepancy between both methods is basically due to the analysis of the distinctive characteristics of each spot separately and not forcing the image processing as a whole, since this is what causes the weakest signals 


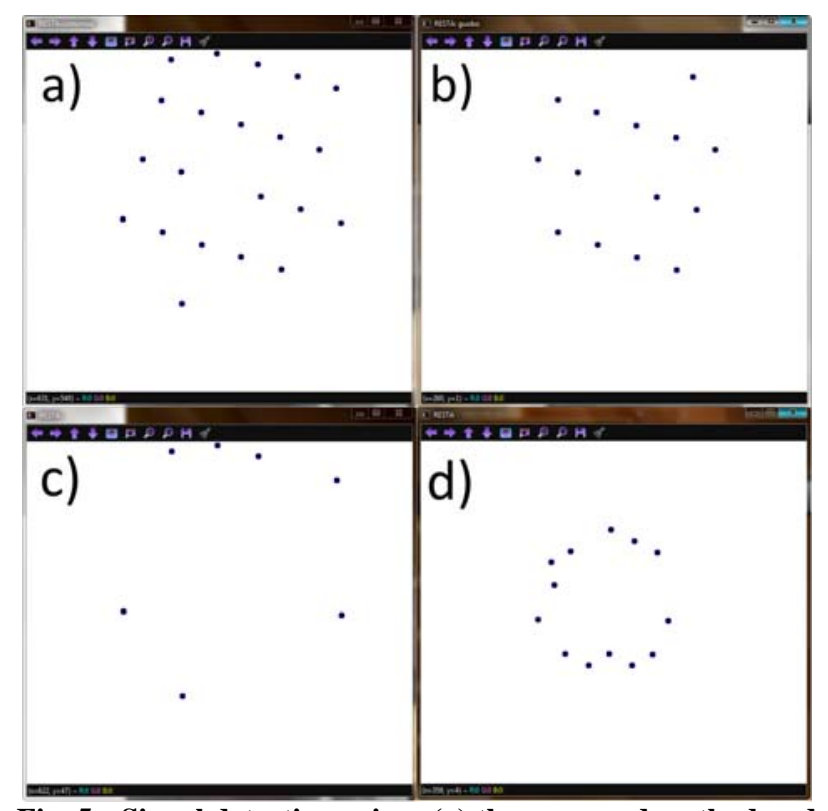

Fig. 5 Signal detection using: (a) the proposed method and (b) the Xie and Lu's algorithm, (c) comparison between both methods through the subtraction of the images $5 a$ and (d) the subtraction result for another diffraction pattern.

to be lost. Table 1 displays the difference between both methods when each one is applied to the selected images. Table 1 registers all the signals discovered, the percentage of signals erroneously detected and the signals that were properly identified by each method.

Table 2 shows the difference of identified signals between both models, indicating the additional percentage of signals detected that the suggested method has in contrast with the one that Xie and $\mathrm{Lu}$ offer.

The result is a statistic with an average of 36 detected signals per diffraction pattern with an error rate of $8.94 \%$ with the Xie and Lu's technique, against an average of 40 identified signals and a $1.60 \%$ error rate with the algorithm suggested. The contrast between both methods is visually presented in Fig. 5, where the negative of the resulting images for both methods were obtained; its results were subtracted by means of OpenCV method subtract() [9] in order to reveal the distinctions in them. Fig. 5c shows as dots the difference between the signals detected by the proposed method (Fig. 5a) and Xie and Lu's work

Table 1 Comparison of the total amount of signals detected by each method, the number of signals correctly identified and the error rate.

\begin{tabular}{|c|c|c|c|c|c|c|}
\hline \multirow[b]{2}{*}{ Pattern } & \multicolumn{2}{|c|}{ Total signals detected } & \multicolumn{2}{|c|}{ Signals detected accurately } & \multicolumn{2}{|c|}{ Signals detected erroneously (\%) } \\
\hline & Xie-Lu & SIPAD & Xie-Lu & SIPAD & Xie-Lu & SIPAD \\
\hline 1 & 20 & 33 & 20 & 33 & 0 & 0 \\
\hline 2 & 38 & 39 & 38 & 39 & 0 & 0 \\
\hline 3 & 14 & 21 & 14 & 21 & 0 & 0 \\
\hline 4 & 36 & 39 & 36 & 39 & 0 & 0 \\
\hline 5 & 98 & 95 & 98 & 95 & 0 & 0 \\
\hline 6 & 63 & 73 & 62 & 72 & 1.58 & 1.37 \\
\hline 7 & 35 & 39 & 35 & 39 & 0 & 0 \\
\hline 8 & 24 & 26 & 24 & 26 & 0 & 0 \\
\hline 9 & 14 & 14 & 14 & 14 & 0 & 0 \\
\hline 10 & 7 & 10 & 7 & 10 & 0 & 0 \\
\hline 11 & 23 & 21 & 23 & 21 & 0 & 0 \\
\hline 12 & 14 & 15 & 14 & 15 & 0 & 0 \\
\hline 13 & 1 & 27 & 0 & 22 & 100 & 18.5 \\
\hline 14 & 37 & 37 & 37 & 37 & 0 & 0 \\
\hline 15 & 46 & 46 & 46 & 46 & 0 & 0 \\
\hline 16 & 52 & 55 & 52 & 55 & 0 & 0 \\
\hline 17 & 35 & 42 & 35 & 42 & 0 & 0 \\
\hline 18 & 47 & 47 & 47 & 47 & 0 & 0 \\
\hline 19 & 130 & 36 & 21 & 31 & 83.9 & 13.9 \\
\hline 20 & 81 & 73 & 79 & 73 & 2.46 & 0 \\
\hline 21 & 56 & 63 & 56 & 63 & 0 & 0 \\
\hline Average & 41.48 & 40.5 & 36.1 & 40 & 8.94 & 1.608 \\
\hline
\end{tabular}


Table 2 Rate of signals identified by SIPAD based on Xie-Lu's method.

\begin{tabular}{ll}
\hline \# Pattern & $\begin{array}{l}\text { Signals found by SIPAD } \\
\text { Compared to Xie-Lu's method (\%) }\end{array}$ \\
\hline 1 & 65.0 \\
2 & 2.6 \\
3 & 50.0 \\
4 & 8.3 \\
5 & -3.1 \\
6 & 16.1 \\
7 & 11.4 \\
8 & 8.3 \\
9 & 0.0 \\
10 & 42.9 \\
11 & -8.7 \\
12 & 7.1 \\
13 & 100.0 \\
14 & 0.0 \\
15 & 0.0 \\
16 & 5.8 \\
17 & 20.0 \\
18 & 0.0 \\
19 & 47.6 \\
20 & -7.6 \\
21 & 12.5 \\
Average & 18.0 \\
\hline
\end{tabular}

(Fig. 5b). Fig. 5d contains the result of a second diffraction pattern processed the same way as Fig. 5c. Clearly, a similar outcome behavior is achieved in these resulting images.

\section{Conclusions}

Using the OpenCV library enables easy implementation of specific image processing and analysis methods; the SIPAD software presented in this paper provides a method for detecting points of interest in an image regardless of whether or not lightning condition varies in different regions of the image or if the signals to detect have a low or high intensity. This is possible because every signal is analyzed in its own environment, therefore, if other methods considered a signal as noise because of the global properties of the image with this technique, every signal of interest is analyzed in a special context. Consequently, most of the points that coincide with the specified characteristics of a signal can be detected even when they are difficult to locate in the original image.

\section{References}

[1] Kalpakjian, S., and Schmid, S. 2002. Manufacturing, Engineering and Technology. México: Pearson Educación.

[2] Orellana, A. M. 2014. “Transmission Electron Microscopy Unit.” University of Malaga. Accessed April 4, 2014. http://www.scai.uma.es/servicios/area_microscopia/tem/t em.html.

[3] Viñas, W. G., and Mancini, H. 2003. Science of Materials. Barcelona: Editorial Ariel.

[4] Esteve, V. 2006. The Rietveld's Method. Castelló de la Plana: Publicacions de la Universitat Jaume.

[5] Goel, A. 2006. Crystallography. New Delhi: Discovery Publishing House.

[6] Borchardt-Ott, W. 2011. Crystallography: An Introduction. Berlin: Springer.

[7] Weirich, T., Lábár, J., and Zou, X. 2004. Electron Crystallography: Novel Approaches for Structure Determination of Nanosized Materials. Erice: Springer.

[8] Nin, G. V., and Echeverría, O. 2000. Introduction to Electron Microscopy Applied to Biological Sciences. Ciudad Universitaria: UNAM.

[9] Bradsky, G., and Kaehler, A. 2008. Learning OpenCV. Sebastopol: O'Reilly.

[10] Sajjad, K. 2014. "Automatic License Plate Recognition Using Python and OpenCV.” M.E.S. College of Engineering, Kuttippuram.

[11] Marcos, A. G., Ascacíbar, F. J. M. de P., Espinoza, A. V., Elías, F. A., Limas, M. C., Meré, J. B. O., and González, E. P. V. 2006. Basic Computer Vision Techniques and Algorithms. España: Universidad de La Rioja.

[12] Xie, G., and Lu, W. 2013. "Image Edge Detection Based on OpenCV.” International Journal of Electronics and Electrical Engineering 1 (2): 104-6.

[13] Lorsakul, A., and Suthakorn, J. 2007. “Traffic Sign Recognition Using Neural Network on OpenCV: Toward Intelligent Vehicle/Driver Assistance System.” Presented at the 4th International Conference on Ubiquitous Robots and Ambient Intelligence, Korea.

[14] Priyankara, G. P. M., De Silva, C. R., Seneviratne, O. W., Silva, R. K. O. H., and Soysa, W. V. D. 2006. "An Extensible Computer Vision Application for Blood Cell Recognition and Analysis.” Department of Computer Science and Engineering.

[15] Zelinsky, A. 2009. "Learning OpenCV-Computer Vision with the OpenCV Library." IEEE Robotics \& Automation Magazine 16 (3): 100-100. 
[16] Yu, Q. C., Cheng, H. H., Cheng, W. W., and Zhou, X. D. 2004. "Ch OpenCV for Interactive Open Architecture Computer Vision.” Advances in Engineering Software 35 (8): 527-36.

[17] Sawyer, L., Grubb, D., and Meyer, G. 2008. Polymer Microscopy. New York: Springer.

[18] Howe, K. D. 2014. A Practical Introduction to Computer Vision with OpenCV. New York: Wiley.
[19] Mainsah, J. A. G. C. E. 2001. Metrology and Properties of Engineering Surfaces. Boston: Kluwer Academic Publishers.

[20] Teutsch, M. B. J. 2012. "Noise Resistant Gradient Calculation and Edge Detection Using LBPs.” Presented at the Computer Vision-ACCV 2012 Workshop.

[21] Laganiere, R. 2011. OpenCV 2 Computer Vision Application Programming Cookbook. Birmingham: Packt Publishing Ltd. 